\title{
Design and development of the ITER CTS diagnostic
}

\author{
Søren B. Korsholm ${ }^{1, *}$, Bruno Gonçalves ${ }^{2}$, Heidi E. Gutierrez ${ }^{1}$, Elsa Henriques ${ }^{4}$, Virginia Infante ${ }^{4}$, Thomas Jensen ${ }^{1}$, \\ Martin Jessen ${ }^{1}$, Esben B. Klinkby ${ }^{3}$, Axel W. Larsen ${ }^{1}$, Frank Leipold ${ }^{1}$, André Lopes ${ }^{2}$, Raul Luis ${ }^{2}$, Volker Naulin ${ }^{1}$, \\ Stefan K. Nielsen ${ }^{1}$, Erik Nonbøl ${ }^{3}$, Jesper Rasmussen ${ }^{1}$, Mirko Salewski ${ }^{1}$, Morten Stejner ${ }^{1}$, Arianna Taormina ${ }^{1}$, \\ Alberto Vale ${ }^{2}$, Catarina Vidal ${ }^{2}$, Laura Sanchez ${ }^{5}$, Raul M. Ballester ${ }^{5}$, and Victor Udintsev ${ }^{6}$, \\ ${ }^{1}$ Technical University of Denmark, Department of Physics, 2800 Kgs. Lyngby, Denmark \\ ${ }^{2}$ Instituto de Plasmas e Fusão Nuclear, Instituto Superior Técnico, Univ. Lisboa, Portugal \\ ${ }^{3}$ Technical University of Denmark, Center for Nuclear Technologies, 4000 Roskilde, Denmark \\ ${ }^{4}$ IDMEC, Instituto Superior Técnico, Univ. Lisboa, Portugal \\ ${ }^{5}$ Fusion for Energy, 08019 Barcelona, Spain \\ ${ }^{6}$ ITER Organisation, 13115 Saint Paul Lez Durance, France
}

\begin{abstract}
The Collective Thomson Scattering (CTS) diagnostic will be a primary diagnostic for measuring the dynamics of the confined fusion born alpha particles in ITER and will be the only diagnostic for alphas below $1.7 \mathrm{MeV}$ [1]. The probe beam of the CTS diagnostic comes from a 60 GHz 1 MW gyrotron operated in a $\sim 100 \mathrm{~Hz}$ modulation sequence. In the plasma, the probing beam will be scattered off fluctuations primarily due to the dynamics of the ions. Seven fixed receiver mirrors will pick up scattered radiation (the CTS signal) from seven measurement volumes along the probe beam covering the cross section of the plasma. The diagnostic is planned to provide a temporal resolution of $\sim 100 \mathrm{~ms}$ and a spatial resolution of $\sim \mathrm{a} / 4$ in the core and $\sim \mathrm{a} / 20$ near the plasma edge where $\mathrm{a}=2.0 \mathrm{~m}$ is the nominal minor radius of ITER. The front-end quasi-optics will be installed in an equatorial port plug (EPP\#12). A particular challenge will be to pass the probing beam through the fundamental electron cyclotron resonance, which is located in the port plug $(\mathrm{R}=10.3 \mathrm{~m})$ for the nominal magnetic field $\mathrm{B}_{\mathrm{t}}=5.3 \mathrm{~T}$. Hence, particular mitigation actions against arcing have to be applied. The status of the design and specific challenges will be discussed.
\end{abstract}

\section{Introduction}

The primary aim of the next step fusion experiment ITER is to demonstrate - in an integrated system - the production of net power from fusing deuterium and tritium. The fusion born alpha particles (birth energy of $3.5 \mathrm{MeV}$ ) will have much higher relative densities than in present machines and will have a substantial effect on the overall dynamics of the ITER fusion plasma. Therefore, diagnosing the dynamics of the fast alpha particles are important, and at the same time challenging. Only a few ITER diagnostics can measure the confined fast alpha dynamics. One of these is the ITER Collective Thomson Scattering (CTS) diagnostic which has the primary role for the fast ion energy spectrum. Recent analysis shows that for alpha energies below $1.7 \mathrm{MeV}$, the CTS diagnostic will be the only diagnostic on ITER [1].

The work towards having a fast ion CTS diagnostic on ITER dates back more than 20 years. Driven by the need for a better diagnostic coverage of the fast ion dynamics in ITER, CTS was included as an enabled diagnostic (more on the implications of 'enabled' later) in the updated ITER baseline in 2007. Scientifically, the inclusion was in part based on the feasibility study of a
CTS diagnostic system for ITER performed at Risø National Laboratory (now DTU - Technical University of Denmark) [2, 3], and also on the advances of the CTS diagnostics on present machines. This article does not intend to give an overview of the present day CTS diagnostics and also not the fast ion CTS principle and analysis. For these, please refer to $[4,5]$.

This paper intends to give a brief overview of the ITER CTS diagnostic, and more particularly the development of the in-vessel front-end components.

\section{Overview of the ITER CTS Diagnostic}

The ITER CTS diagnostic - as proposed in the feasibility study [2, 3] - operates in the $60 \mathrm{GHz}$ frequency range, which for the nominal ITER magnetic field of $\mathrm{B}_{0}=5.3 \mathrm{~T}$ is below the fundamental $\mathrm{EC}$ resonance in the plasma. The probing beam injected in $\mathrm{X}$-mode is generated by a $1 \mathrm{MW} 60 \mathrm{GHz}$ gyrotron. The frequency range of the scattered radiation will be from 55 to $65 \mathrm{GHz}$. The expected temporal resolution will be $100 \mathrm{~ms}$ and the spatial resolution will be in the order of $\mathrm{a} / 4$ in the center, where $\mathrm{a}=2.0 \mathrm{~m}$ is the nominal minor radius of ITER. The spatial resolution varies with plasma scenario and location of measurement volume. For the

* Corresponding author: sbko@fysik.dtu.dk 
reference scenario (Scenario 1 - ITER Baseline Scenario) the resolution will vary from $\sim \mathrm{a} / 2$ on the high field side to $\sim \mathrm{a} / 30$ on the low field side near the edge. The CTS system will have seven measurement volumes in the plasma.

The ITER CTS diagnostic is an 'enabled' diagnostic in the ITER baseline. The consequence is that primarily the in-vessel components and captive components that cannot be retrofitted after first plasma are part of the credited construction baseline. Fusion for Energy (F4E) will be responsible for delivering the in-vessel front-end components, as well as the functional specifications of the rest of the system. The responsibility for the design and delivery of the ex-vessel components (including the vacuum vessel windows) of the CTS diagnostic is under ITER IO. The different components of the CTS diagnostic are presented in the schematic in Figure 1. The present paper will focus on the design and development on the front-end components, i.e. the invessel components (in the white box in the left of the figure).

The in-vessel CTS components will be installed in a separate drawer \#3 in equatorial port plug \#12. A drawer consists of a diagnostic shield module (DSM) and a diagnostic first wall (DFW). The gyrotron will be installed in a future enclosure in the assembly hall and the matching optics unit (MOU) box will include a polarizer set to ensure X-mode injection in the plasma. Further, it will be connected to the port plug by a $\sim 110 \mathrm{~m}$ transmission line consisting of circular corrugated waveguide and mitre bends. At the closure plate, the gyrotron radiation is coupled through a $\varnothing 110 \mathrm{~mm}$ inner diameter (ID) CVD (Chemical Vapour Deposition) diamond window by a set of two ex-vessel coupling mirrors. The ex-vessel transmission of nine identical receiver lines will be through circular corrugated waveguides connecting the $\sim 37$ meters to the diagnostic building where the receiver electronics will be located.

The number of receiver lines was limited by two factors: possible space allocation along the path between the port cell and the diagnostic building - limit nine waveguides of ID $63.5 \mathrm{~mm}$ - and the space allocation on the closure plate. Each vacuum window takes up space for the actual window, the window assembly, and the zones for installation and maintenance. In case an individual window was used for each receiver transmission line, this would have meant that no more than five receiver windows would fit - and similarly only five transmission lines could leave the portplug. Hence, it was proposed to put three large (Ø160 mm ID) windows that could be used commonly by several receiver lines. The quasi-optical paths, designed at DTU, could fit three quasi-optical lines through each window assembly using a set of two in-vessel and two ex-vessel coupling mirrors for each line. In this way the maximum number of transmission lines between the diagnostic building and the vacuum vessel was set to nine. The geometry of the closure plate is illustrated in Figure 2.

The current status of the CTS diagnostic development process is that the Conceptual Design Review was conducted and passed by ITER IO in 2013. The F4E framework partnership agreement contract with the DTU/IST consortium was initiated in 2014 and the Intermediate Design Review (or Pre Annex B Design Review) was conducted and passed in 2017. Currently, the design of the in-vessel system is in the preparatory

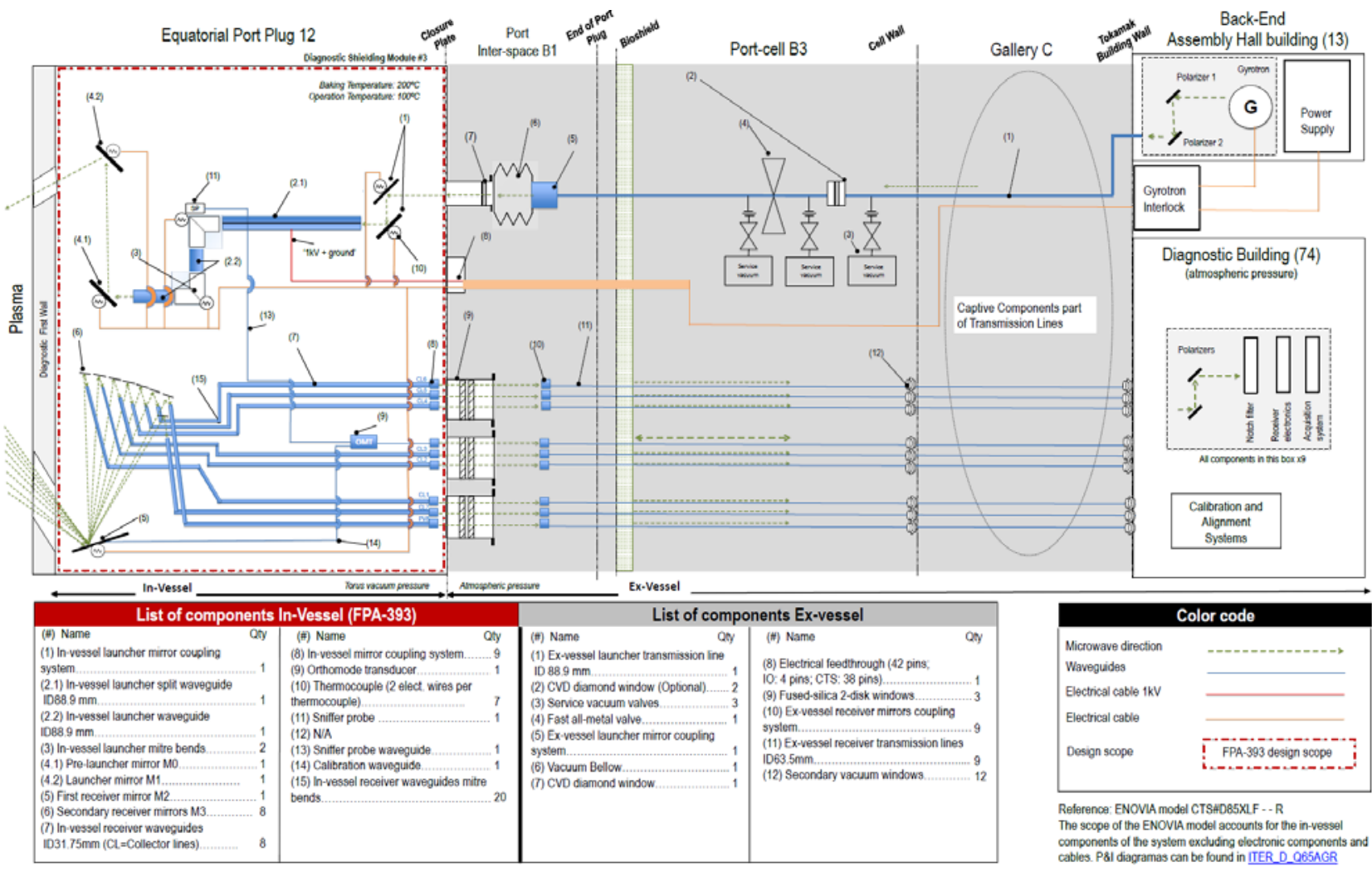

Fig. 1. Schematic for the ITER CTS diagnostic. 
design phase, and the Preliminary Design Review for the full system is expected within 12 months.

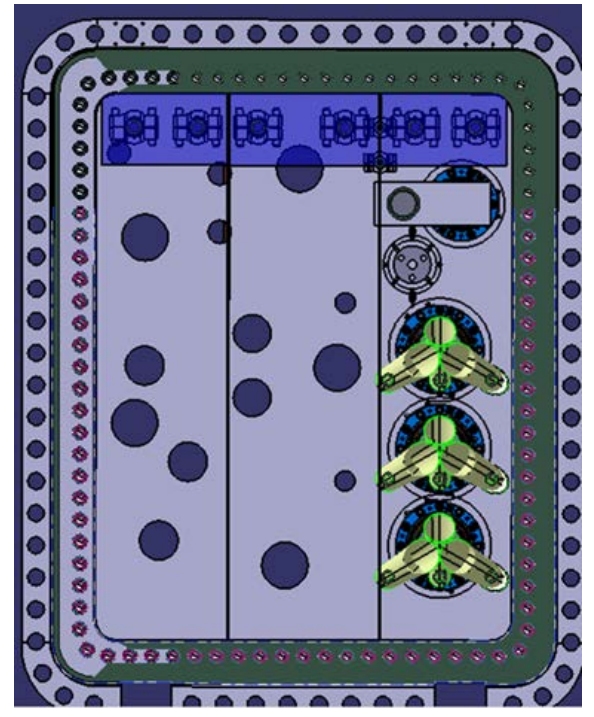

Fig. 2. CAD drawing of the closure plate of equatorial port plug \#12 seen from outside the vacuum vessel. DSM \#3 is to the right. The launcher line window (Ø110mm CVD) is in the top and below are the three receiver windows $(\varnothing 160 \mathrm{~mm}$ fused silica double disk).

Prior to the Intermediate Design Review, a series of analysis and reports were performed and presented for the system design at that time. This included e.g. diagnostic performance analysis, critical component analysis and design, prototype needs analysis and plans, Reliability, Availability, Maintainability and Inspectability (RAMI) analysis, thermomechanical analysis (to be included in structural integrity analysis), remote handling analysis, neutronics analysis Some of the analyses have been published, like e.g. the RAMI analysis [6] and the neutronics modelling results [7].

\section{CTS front-end component design}

The in-vessel parts of the ITER CTS diagnostic are exclusively located in drawer \#3 of the remote handling and first plasma equatorial port plug \#12. As any invessel ITER system the design is restricted by a long list of interfaces and requirements; and in addition some more internal challenges. A full account of these is given in the documentation for the Intermediate Design Review. In this paper we shall only briefly describe some of the major issues.

An overall challenge for the design has been the requirement to have no moveable parts, while ensuring diagnostic performance and still having some margin or flexibility to different plasma scenarios. At the same time the quasi-optical sizes of components (at $60 \mathrm{GHz}$ ) and required apertures are important for diagnostic performance, while large cut-outs in the DSM and the DFW are challenging from a neutronics and thermomechanical point of view. Particularly the cut-out for the launcher beam in the DFW is a compromise between diagnostic performance (larger cut-out could focus the beam better in the plasma and increase spatial resolution) and (neutronics) shutdown dose rate at the closure plate.

As mentioned in the section above, the limitations at the closure plate (and beyond) limited the number of transmission lines from the vessel. The number of channels to the diagnostic building is nine, and they have been allocated with seven actual receiver lines, one passive view, and one line for a calibration/sniffer probe. Present experiments on e.g. ASDEX Upgrade [8] have shown that events in the plasma contribute to the background signal in the CTS measurements, in a way that is not properly captured by the normal ECE background subtraction. Therefore, the introduction of a passive view can help diagnosing these parts of the signal and improve the data analysis significantly. The passive view is a receiver line as the other seven receiver lines, with the important exception that there is no overlap between the gyrotron probing beam and the passive view beam. At the same time, for the passive view, the beam geometry and the plasma of view should be as similar as possible to the active receiver views.

The seven receivers observe the gyrotron probe beam at seven different radial locations. In this way, the CTS diagnostic can provide a radial profile of the fast ion measurements despite not having any moveable parts. As it can be seen in Figure 3, all seven receiver beams (as well as the passive view) share the common plasma facing receiver mirror in the bottom of the drawer. From there each receiver line has a separate secondary receiver mirror that couples the received signal into circular corrugated waveguides of $\varnothing 31.75 \mathrm{~mm}$. The transmission lines continue to the mirror coupling system at near the closure plate via waveguides and three mitre bends per line.

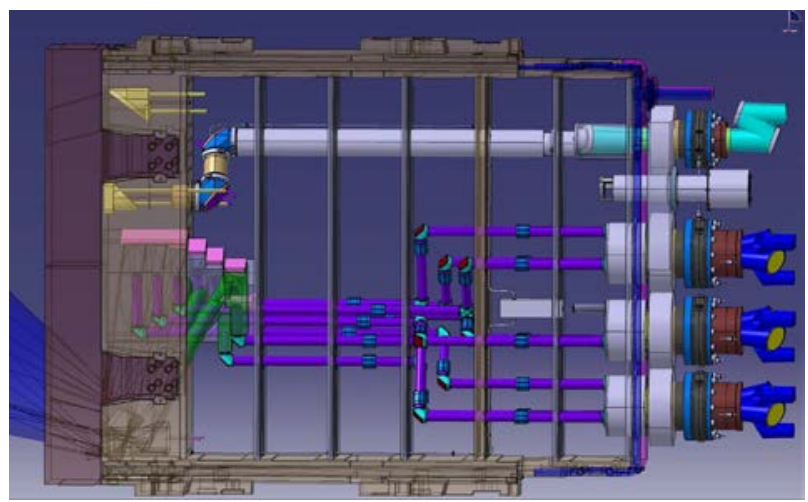

Fig. 3. CAD drawings of ITER CTS diagnostic in drawer \#3 of equatorial port plug \#12.

The last of the nine transmission lines are reserved for the double use as a gyrotron transmission line sniffer probe during diagnostic operation and as a receiver calibration source transmission line during maintenance. This can be done as the two functionalities will never be done simultaneously and in this way we saved one transmission line through the closure plate. It has been enabled by an in-vessel passive switching system using directional couplers and orthomode transducers ensuring sufficient isolation $(>30 \mathrm{~dB})$ between the two uses. The 
sniffer probe will be installed in the first in-vessel mitre bend (seen in the direction of the gyrotron probe beam) and it will observe the long launcher waveguide to be described below. The calibration waveguide will be connected to a small hole in the center of the common receiver mirror and will emit microwaves in a uniform pattern that will be picked up by the individual secondary receiver mirrors in order to calibrate the receiver transmission lines. The calibration source will be located in the diagnostic building and can be controlled with broadband signal or sweeping signal and can naturally also be varied in power level as appropriate.

The primary challenge of the ITER CTS diagnostic in the port plug is the transmission of the gyrotron probe beam through the fundamental electron cyclotron resonance, which is located inside the port plug for most of the proposed operating main magnetic fields of ITER. For the nominal magnetic field of $5.3 \mathrm{~T}$ the fundamental resonance for $60 \mathrm{GHz}(\mathrm{B}=2.14 \mathrm{~T})$ is at a major radius of $\mathrm{R}=10.4 \mathrm{~m}$. The location of the resonance will naturally move with different operating magnetic fields (e.g. for $\mathrm{B}_{0}=3.5 \mathrm{~T}$ the resonance is at $\mathrm{R}=9.6 \mathrm{~m}$ ).

In order to limit the risk of stray light and in order to limit the required cut-outs in the DSM, the design solution has been to use waveguide rather than quasioptical transmission via mirrors through the DSM. The chosen inner diameter of the circular corrugated waveguide is $88.9 \mathrm{~mm}$. Transmitting a $1 \mathrm{MW}$ probe beam through this waveguide and passing the fundamental resonance at an uncontrolled ambient pressure constitutes a genuine risk for creating a plasma breakdown inside the waveguide. This would hamper the diagnostic capability and in the worst case lead to partial or full absorption of the $1 \mathrm{MW}$ beam in the waveguide. For this reason, mitigation mechanisms have to be implemented. Several mitigation actions have been considered. Presently, the most developed mitigation action is adapting a split biased waveguide. This solution has previously been used at DITE and DIII-D. In short, the launcher waveguide is split along its axis into two halves. They are isolated from each other (and the surroundings), and one half is grounded, while the other is connected to a bias voltage of $+1 \mathrm{kV}$. In case the gyrotron probe beam ionises a neutral atom or accelerates a free electron, the sweep time (time from waveguide center to the biased wall) is much shorter than the gyration time. In this way, the risk of a breakdown should be mitigated. As a side effect, the biased waveguide will act as a diagnostic for such electron sweeping events; as such an event will generate a current.

Some of the quasi-optical components have been prototyped and tested in the DTU microwave laboratory in order to verify the quasi optical codes and mirror designs. The prototypes of the mirrors were produced by 3D plastic printing at DTU. Following this, the mirror surfaces were covered by metal foil. This method has been used and tested before on prototyping of quasi optical components.

An example can be seen in Figure 4 and 5 below. This prototype was designed to test the receiver transmission line coupling system at the closure plate. The tests comprised of verification of the mirror and beam shapes - verifying the codes and design - and confirmation that the in-vessel cylinder around the mirrors (mitigating stray light) and the vacuum window assembly "tunnel" do not give rise to interference.

The results of the prototype tests (see example in Figure 6) verify that the designed quasi-optical components (in particular the mirrors) for the ITER CTS transmission line are performing as expected and have an acceptable correspondence to the modelling. The next step prototypes include 3D plastic printing of circular corrugated waveguides. These will then have a conducting coating applied by either conducting spray paint or other external coating methods.

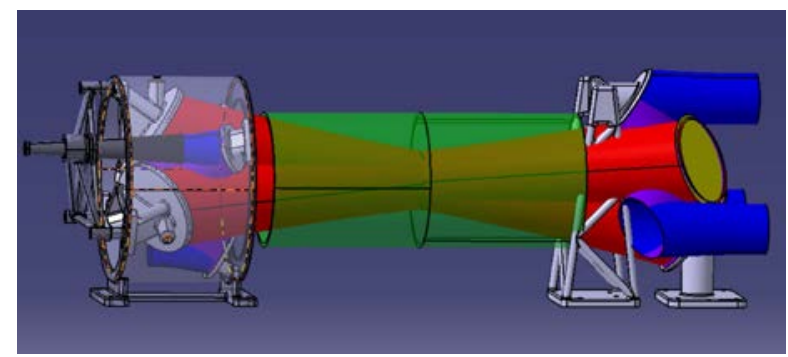

Fig. 4. CAD drawings of the ITER CTS prototypes of the closure plate coupling system for three receiver beams. The green duct represents the $400 \mathrm{~mm}$ long vacuum window assembly.

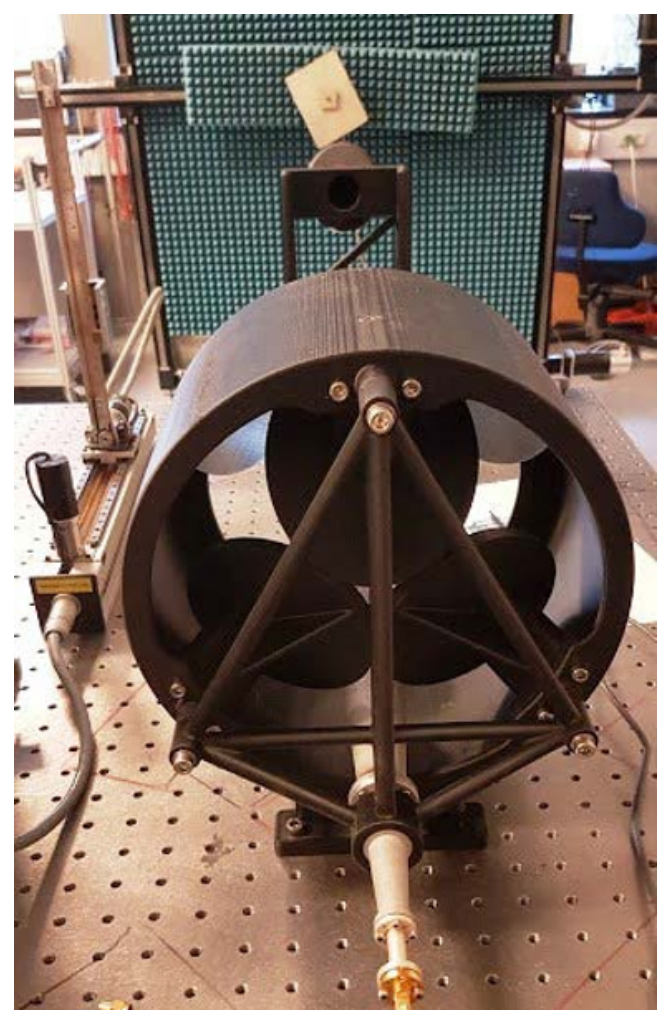

Fig. 5. The prototype testing setup in the DTU microwave lab of the receiver line coupling mirror units as presented in the CAD model in Figure 4. 


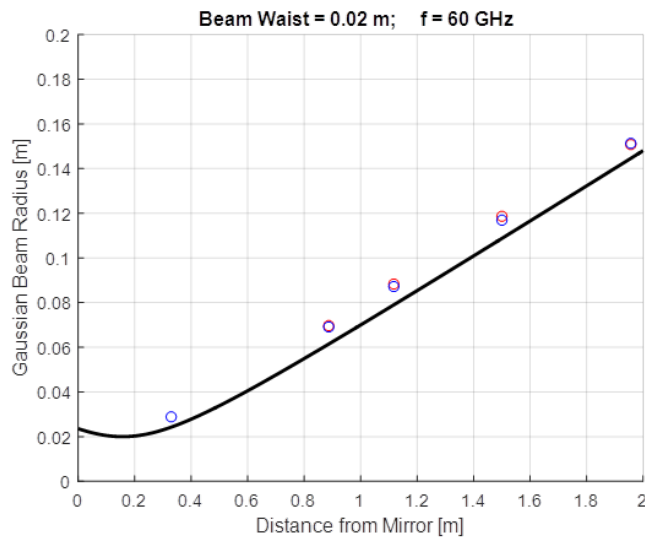

Fig. 6. Results of the prototype testing of the receiver transmission line coupling mirrors at the closure plate (shown in Figure 4 and 5). The solid black line shows the calculated (desired) beam radius and the red (blue) dots show the measurements without (with) the vacuum window assembly "tunnel". (1.0 x Gaussian width). The entrance of the waveguide is located at the position of the beam waist $(0.157 \mathrm{~m}$ from the mirror).

\section{Measurement capabilities}

The present design of the ITER CTS diagnostic as presented above does not have any moveable parts, and thus the paths of the seven primary receiver beams are fixed. The probing frequency of $60 \mathrm{GHz}$ was chosen as a compromise between the acceptable background ECE levels, maximum obtainable signals, and spatial resolution. For $60 \mathrm{GHz}$ the O-mode cut-off is at the edge of the plasma, hence $\mathrm{X}$-mode injection is required. However, the $\mathrm{X}$-mode R-cut-off is quite close to the receiver frequency range, so depending on the plasma scenario (primarily the density profile) and the angle of injection (to the density gradient), refraction of the microwave beams can be significant. This is illustrated by the ray tracing results in Figure 7 . It is seen that refraction is most significant for the outermost receiver beams having the largest poloidal angle to the density gradient. The positive side effect of this is a more perpendicular incidence on the probing beam, and hence an increased spatial resolution in the edge, i.e. a/20 $\mathrm{a} / 30$, where a is the plasma minor radius. However, the fast ion density for these scattering volumes is presumably very low. The refraction in the poloidal plane will thus be different for various plasma scenarios and density profiles, and optimisation of the fixed mirror geometry will have to be based on a few reference scenarios and optimised for some robustness.

The probe and receiver beams do also have a toroidal injection angle in order to have a resolved angle $\varphi=\angle\left(\mathrm{k}^{\delta}, \mathrm{B}\right)$ that is at least 5 degrees from 90 degrees in order to avoid interference in the CTS spectrum from plasma waves propagating perpendicularly to the magnetic field. $\varphi$ is the angle between the main magnetic field $\mathrm{B}$ and the wave vector of the resolved fluctuations $\mathrm{k}^{\delta}$. The (slightly) toroidal injection angles do also give rise to refraction in the toroidal direction, which will depend on plasma scenarios and density profiles. However, the toroidal refraction is similar for the probe and receiver beams, and hence the overlaps between them should not be lost.

In the following we define the spatial resolution as the extent of the region from which $75 \%$ of the scattered signal originates.

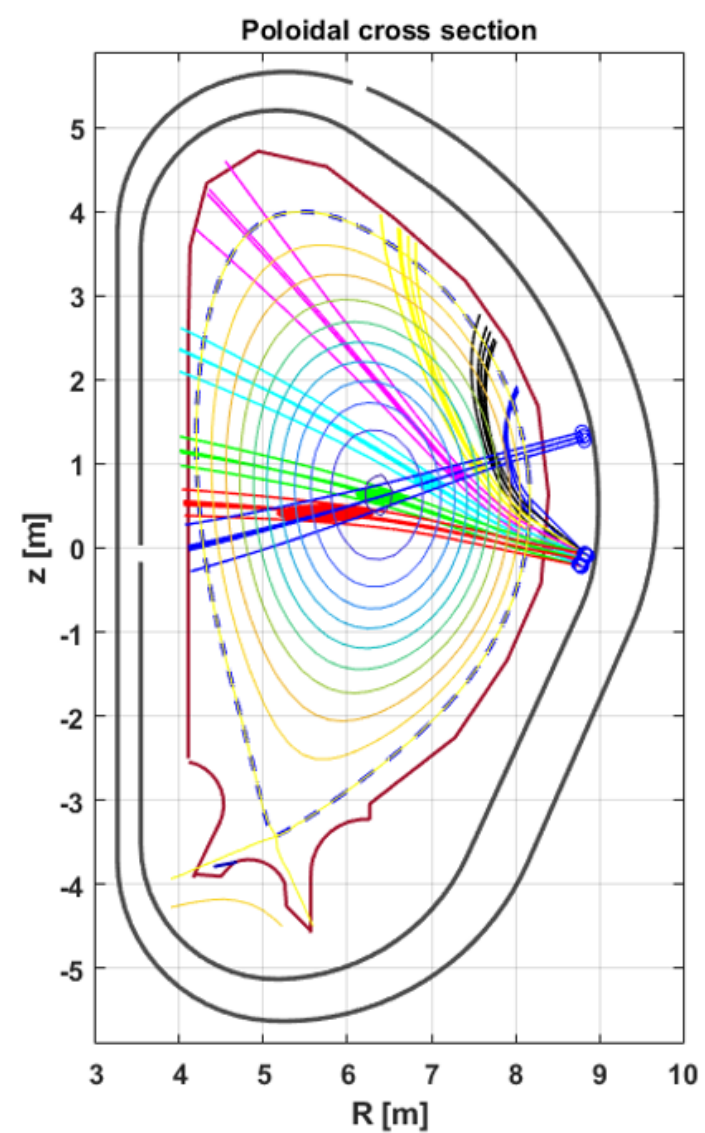

Fig. 7. Poloidal view of the ray tracing results for the ITER CTS probe and the seven receiver beams. The equilibrium is for Scenario 1 (reference scenario) and has been provided by F4E. The electron density and temperature profiles for the scenario are presented in Figure 8.

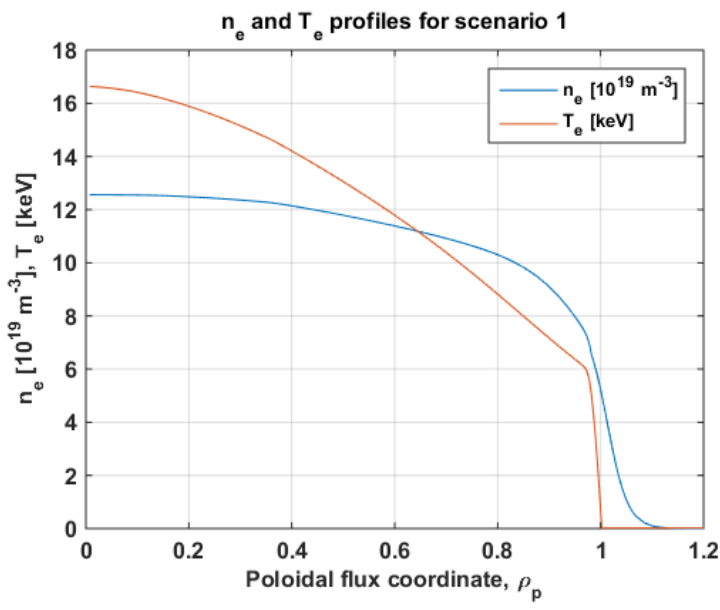

Fig. 8. Electron density and temperature profiles for the provided Scenario 1 (ITER Baseline Scenario) that was used for the ray tracing presented in Figure 7. 
For the presently provided reference Scenario $1^{\mathrm{a}}$, the ITER Baseline Scenario (see Figure 8), the spatial resolution for the receiver observing the high field side is $\sim \mathrm{a} / 2$ and for the center of the plasma it is $\sim \mathrm{a} / 4$, and then increasing towards $\sim \mathrm{a} / 30$ near the low field side plasma edge.

The study of the potential measurement capabilities will be reported in a separate publication, and the extent of the study is too comprehensive to fully review here. However, the conclusion is that the ITER CTS diagnostic can provide measurements of the projected 1D fast-ion velocity density function of the confined alphas and fast ions, as well as the alpha fast ion density (under isotropy assumptions) with a temporal resolution of $100 \mathrm{~ms}$ and within the required statistical uncertainty of $20 \%$. A series of fitting tests with synthetic data has also shown that the present CTS system setup and analysis codes can reproduce the true alpha density used in the tests with only small discrepancies.

CTS has also been assigned a supplementary role for measuring the bulk ion temperature profile as well as the $\mathrm{n}_{\mathrm{H}} / \mathrm{n}_{\mathrm{D}}$ and the fuel ion ratio $\mathrm{n}_{\mathrm{T}} / \mathrm{n}_{\mathrm{D}}$ in the plasma core. As presented in $[9,10]$ the two latter will require a rather perpendicular scattering geometry, i.e. $\varphi=\angle\left(\mathrm{k}^{\delta}, \mathrm{B}\right) \sim$ near 90 degrees. As the CTS mirror geometry is fixed, and deliberately off-perpendicular as described above, the ideal setup for fuel ion and isotope ratio measurements cannot be achieved. Thus these measurements are not design driving for the ITER CTS diagnostic. However, for a given plasma scenario and scattering spectrum, it may be possible to infer some information relating to the fuel ion ratio, as there will be spectral content containing information on this even with the off-perpendicular geometry. However, integrated data analysis accounting for other measurements may be required. In the analysis of the CTS spectrum with the aim of getting the information on the fast ion dynamics, the analysis will additionally provide some information on the bulk ion temperature and the ion rotation velocity, and may thus contribute to the determination of these via integrated data analysis.

The performance analysis results presented briefly above are based on the (conservative) assumption of a background ECE level of $100 \mathrm{eV}$. Since the CTS frequency range is far below the (cold) fundamental resonance the estimation of ECE background levels are not straightforward. Some modelling results predict virtually $0 \mathrm{eV}$, but high temperatures, multiple reflections of the beams on the vessel walls and Doppler shift of the resonance will give rise to a non-zero ECE level. While still searching for a good method/code for prediction of the level, we have assumed $100 \mathrm{eV}$.

Presently, no official full data sets of ITER plasma scenarios, plasma parameters and profiles, fast ion densities etc. exist. We have assembled the required information from sources at ITER IO and F4E. We follow closely the development of the official and traceable sets of ITER plasma scenarios and parameters

\footnotetext{
a See last paragraph of Section 4 concerning lack of traceability of plasma scenario data
}

performed in the IMAS project at ITER IO, and will update our analysis and predictions based on this data.

\section{5 conclusions}

The present paper has intended to give an overview of the present status of the development of the ITER CTS diagnostic. It is in no way exhaustive of the extensive work done on a long range of topics for both the invessel and the ex-vessel parts of the system. Specific publications on these are planned and underway.

We have presented that the ITER CTS diagnostic is in the preparatory design phase, and the Preliminary Design Review for the system is expected within 12 months.

The diagnostic will be installed in drawer \#3 of Equatorial Port Plug \#12 and will consist of robust nonmoveable quasi-optical parts. Based on modelling, the diagnostic is expected to fulfil its primary role for measuring the fast alpha and fast ion dynamics in seven measurement volumes in the plasma.

\section{Acknowledgement}

The work leading to this publication has been funded partially by Fusion for Energy under the Framework Partnership Agreement F4E-FPA-393. This publication reflects the views only of the author, and Fusion for Energy cannot be held responsible for any use which may be made of the information contained therein. The views and opinions expressed herein do not necessarily reflect those of the ITER Organization.

\section{References}

1. M. Salewski, et al., Alpha-particle velocity-space diagnostic in ITER, submitted to Nucl. Fusion, (2018)

2. H. Bindslev, F. Meo and S.B. Korsholm, ITER Fast Ion Collective Thomson Scattering, Feasibility study and conceptual design, EFDA Report (2003)

3. H. Bindslev, et al, Rev. Sci. Instrum, 75, 3598 (2004)

4. S.B. Korsholm et al, Nucl. Instr. Meth. Phys. Res. A, 623, 677 (2010)

5. S.K. Nielsen, et al, Phys Scr, 92, 024001 (2016)

6. V. Infante, et al, Fus. Eng Design, 123, 663 (2017) (http://dx.doi.org/10.1016/j.fusengdes.2017.05.001)

7. A. Lopes, et al, Neutronics analysis of the ITER Collective Thomson Scattering System, submitted to Fus. Eng. Des. (2018)

8. S.K. Nielsen, et al, Plasma Phys. Control. Fusion 57 035009 (2015)

9. S.B. Korsholm et al, Phys. Rev. Lett. 106165004 (2011)

(http://dx.doi.org/10.1103/PhysRevLett.106.165004)

10. M. Stejner et al, Plasma Phys. Control. Fusion 53 065020 (2011) 